\title{
Caracterização dos agregados em solos sob cultivo no Cerrado, MS
}

\section{Aggregates characterization of soils under cultivation in Cerrado, $\mathbf{M S}^{1}$}

\author{
Itaynara Batista ${ }^{2}$; Maria Elizabeth Fernandes Correia ${ }^{3}$; Marcos Gervasio Pereira ${ }^{4 *}$; \\ Wanderlei Bieluczyk ${ }^{5}$; Jolimar Antônio Schiavo ${ }^{6}$; Nilvânia Aparecida de Mello ${ }^{7}$
}

Resumo

Impactos sobre o ambiente do solo e a atividade biológica resultam em alterações nos processos de formação de agregados biogênicos e fisiogênicos. Com o objetivo de avaliar a influência física e biológica na gênese de agregados dos solos do Cerrado, bem como determinar quais os principais fatores do ambiente que determinam as características dos agregados em área de produção sob sistema de integração lavoura-pecuária (ILP), foi desenvolvido esse trabalho na Fazenda Cabeceira, MaracajuMS, em áreas de ILP. As áreas avaliadas foram: Cerradão, pasto/milho, milho/algodão e algodão/soja, sendo realizadas avaliações nas épocas seca (maio/2009) e chuvosa (março/2010). Para identificação das vias de agregação foram utilizados padrões morfológicos, sendo estabelecidos três tipos de agregados: fisiogênicos, biogênicos e intermediários. Os agregados foram analisados quanto ao complexo sortivo, carbono e estabilidade, o solo foi analisado quanto o complexo sortivo, fracionamento granulométrico da matéria orgânica do solo, frações oxidáveis do carbono orgânico total, análise granulométrica e macrofauna edáfica. Em todas as áreas estudadas, na época seca, foram quantificados os maiores valores de agregados intermediários, enquanto na época chuvosa, de maneira geral, não foram observadas diferenças dos agregados formados pelas diferentes vias nas áreas, exceto algodão/soja. Os agregados biogênicos apresentaram correlação positiva com teores de carbono e foram encontrados em menor quantidade em comparação aos fisiogênicos e intermediários. Os diferentes tipos de agregados formados, além de apresentarem características morfológicas diferentes, também apresentam diferenças quanto às características químicas.

Palavras-chave: Agregação do solo, matéria orgânica do solo, manejo do solo

\begin{abstract}
Impacts on the environment and soil biological activity result in changes in the processes of formation of biogenic and fisiogenic aggregates. Aiming to evaluate the influence on physical and biological genesis of aggregates of Cerrado soils and determine the main environmental factors that determine the characteristics of households in the area of production under integrated crop-livestock (ICL), was developed this study in Farm Cabeceira, Maracaju-MS, in areas of ICL. The areas evaluated were:
\end{abstract}

${ }^{1}$ Parte da dissertação de mestrado da primeira autora apresentada ao Curso de Pós-Graduação em Agronomia, Ciência do Solo (CPGA-CS) da Universidade Federal Rural do Rio de Janeiro. URRJ, Rio de Janeiro. Apoio CNPq.

${ }^{2}$ Discente de Doutorado em Agronomia (Agricultura), Universidade Estadual Paulista, Faculdade de Ciências Agronômicas, Campus de Botucatu, UNESP, Botucatu, SP. E-mail: itaynarabatista@yahoo.com.br

${ }^{3}$ Pesquisadora da Embrapa Agrobiologia. BR 465, km 7, CEP 23890-000, Seropédica, RJ. E-mail: elizabeth.correia@embrapa.br

${ }^{4}$ Prof. Associado IV do Dept ${ }^{\circ}$ de Solos da UFRRJ BR 465, km 7, CEP 23890-000, Seropédica, RJ. E-mail: gervasio@ufrrj.br

${ }_{5}^{5}$ Discente de Mestrado do CPGA-CS, Dept ${ }^{\circ}$ de Solos, UFRRJ, BR 465, km 7, CEP 23890-000, Seropédica, RJ. E-mail: wanderleibieluczyk@gmail.com

${ }^{6}$ Prof. Adjunto IV da Universidade Estadual de Mato Grosso do Sul, UEMS, Rod. Aquidauana, km 12, CEP 79200-000 Aquidauana, MS. E-mail: schiavo@uems.br

${ }^{7}$ Prof ${ }^{a}$ da Universidade Tecnológica Federal do Paraná, UTFPR, Via do Conhecimento, km 1 CEP 85503-390, Pato Branco, PR. E-mail: nilvania@utfpr.edu.br

* Autor para correspondência 
Savana, pasture/maize, corn/cotton and cotton/soybeans being evaluated during the dry (May/2009) and rainy (March 2010) season. To identify the pathways of aggregation were used morphological patterns, and established three clusters: fisiogenic, biogenic and intermediates. The aggregates were analyzed for exchangeable cations, carbon and aggregate stability, soil was analyzed for the exchangeable cations, particle size fractionation of soil organic matter, oxidizable fractions of total organic carbon, particle size analysis and soil macrofauna. In all areas studied, in the dry season, the highest values were quantified aggregate intermediates, while in the rainy season, in general, no differences were observed aggregates formed by different routes in areas except cotton/soybeans. The aggregates showed positive correlation with biogenic carbon and were found in lesser amounts compared to fisiogenic and intermediates. The different types of aggregates formed, besides having different characteristics morphological also differ as to chemical characteristics.

Key words: Soil aggregation, organic matter, soil management

\section{Introdução}

O Cerrado é o segundo maior bioma brasileiro, menor apenas que o bioma Amazônico. Originalmente ocupava $23,9 \%$ do território nacional e, em 2010, com os desmatamentos, passou a representar 12,4\% (IBGE, 2010).

As transformações de uso da terra no Cerrado tiveram início na década de 70 . Juntamente com o desmatamento, algumas técnicas inadequadas de manejo do solo propiciaram a rápida degradação, ocasionando perdas de matéria orgânica e nutrientes (RESCK et al., 2008).

O sistema Integração Lavoura-Pecuária (ILP) surgiu como uma alternativa de produção conservacionista, porém mais complexa, com o objetivo de manter a produtividade agrícola e indiretamente promover a recuperação e renovação de pastagens. Nesse sistema, a introdução da lavoura não é eventual, mas parte constante de um planejamento de produção de grãos e de produção animal, que interage e se completa biológica e economicamente (MACEDO, 2001).

O ILP possibilita melhorias na qualidade física do solo, pois, a inclusão de pastagens na rotação de culturas pode aumentar rapidamente a estabilidade dos agregados, a macroporosidade e a condutividade hidráulica, devido à combinação da ausência de preparo durante o ciclo da pastagem, presença de um denso sistema radicular que atua como agente agregante e maior atividade da macrofauna do solo na fase de pastagem (MARCHÃO, 2007).
Em estudo realizado por Salton (2005), o autor observou que a organização da estrutura do solo mostrou-se intimamente ligada ao maior aporte de $\mathrm{C}$ ao solo, que ocorreu de forma mais expressiva com a presença de pastagens.

O processo de agregação, que tem início com a união das partículas unitárias, pela ação de inúmeros ciclos biológicos, físicos e químicos (TISDAL; OADES, 1982), é dependente de diversos fatores. Os principais são: tipo e teor de argila, metais polivalentes, óxidos e hidróxidos de ferro, alumínio e manganês, exsudatos orgânicos de plantas, substâncias orgânicas provenientes da ação de microrganismos e outros compostos orgânicos. A matéria orgânica, os óxidos e os hidróxidos são agentes cimentantes, tanto das partículas unitárias quanto das partículas secundárias (agregados) do solo. Podem-se incluir, também, os ciclos de umedecimento e secagem como importante fator ambiental que interfere na agregação do solo (CARVALHO, 1991; MALTONI, 1994 apud BASTOS et al., 2005).

A formação de agregados no solo a partir da influência dos fatores citados constitui via lenta de formação, sendo esses denominados de agregados fisiogênicos, ou seja, formados por ações físicas, químicas e adição de matéria orgânica. Outra via de formação de agregados no solo é a denominada de via biogênica, onde os agregados são formados pelas fezes produzidas por indivíduos da fauna do solo, essa via de formação é mais rápida em comparação à primeira (MELLO; CÉCILLON; BRUN, 2008). 
Ainda no aspecto biológico, as raízes têm papel de destaque, pois em paralelo ao aumento de sua densidade e comprimento, verificam-se os maiores valores de agregação, sendo que raízes fibrosas produzem elevados níveis de macroagregados. O aumento da diversidade e quantidade de flora e fauna do solo são importantes para melhorar a agregação, sendo a atividade da fauna edáfica importante na formação de complexos organo-minerais e na agregação (BRONICK; LAL, 2005).

As estruturas biogênicas, tais como agregados, poros e galerias de várias formas e tamanhos são habitats de outros organismos. Há evidências de que macroagregados biogênicos são microsítios importantes para a formação de microagregados e, conseqüentemente, para a estabilização da matéria orgânica do solo (MOS) e o armazenamento de nutrientes, que são liberados lentamente sobre a sua degradação (BRUSSAARD et al., 2007).

Diversos estudos tem sido realizados para avaliar a contribuição da macrofauna edáfica na dinâmica dessas estruturas (DECAËNS; GALVIS; AMÉZQUITA, 2001; JONGMANS; PULLEMAN; MARINISSEN, 2001; JONGMANS et al., 2003; PULLEMAN; MARINISSEN, 2004; PULLEMAN et al., 2005; VELASQUEZ et al., 2007) e segundo Ingham (2000) a composição dos organismos do solo é influenciada pela sazonalidade e dependente da fonte de alimentação, portanto podem apresentar composições diferentes em ambientes distintos como florestas, cultivos e pastagens podendo beneficiar ou afetar a cadeia.

Em relação à interação entre organismos e manejo de solo, Pulleman e Marinissen (2004), relatam que, impactos sobre o ambiente do solo e a atividade biológica resultam em alterações nos processos de formação de agregados biogênicos e fisiogênicos. Dependendo do manejo agrícola, a agregação do solo pode fornecer proteção física contra a rápida decomposição da MOS e que de acordo com o tipo de solo e o sistema de manejo adotado, pode influenciar a qualidade e a quantidade de insumos orgânicos.
Em relação ao Bioma Cerrado, se tornam necessários estudos sobre a qualidade biológica e estrutural, visto a importância desse bioma no cenário nacional de biodiversidade e de produção agrícola. Estudos de agregação nesse bioma têm sido realizados, avaliando principalmente as diferenças entre os sistemas de manejo, contudo, poucos foram realizados com informação sobre a contribuição biológica na estrutura do solo (REATTO et al., 2009; VOLLAND-TUDURI, 2005; BALBINO et al., 2002). Diante do exposto, os objetivos deste trabalho foram: avaliar a influência física e biológica na gênese de agregados dos solos do Cerrado, bem como determinar quais os principais fatores do ambiente que determinam as características dos agregados em área de produção sob sistema ILP.

\section{Material e Métodos}

A área de estudo localiza-se na Fazenda Cabeceira, Maracaju - MS, em latitude 21³6'52" sul e longitude $55^{\circ} 10^{\prime} 06^{\prime \prime}$ oeste, em uma altitude de 384 metros, situando-se a $160 \mathrm{~km}$ da capital do estado. O clima da região é tropical com temperaturas mínimas de $15^{\circ} \mathrm{C}$ e máximas de $33^{\circ} \mathrm{C}$, a precipitação média anual oscila entre 1300 a 1500 $\mathrm{mm}$.

As amostragens foram realizadas na Fazenda Cabeceira, que foi uma das pioneiras na implantação da ILP no Cerrado em 1989, sendo inicialmente introduzida a rotação de soja com pastagem e em 2003 implantado o sistema que funciona atualmente, pastagem, soja e algodão como culturas de verão e pastagem, milho+braquiária e aveia+pastagem como culturas de inverno. As áreas possuem o seguinte histórico: soja/milho+braquiária/algodão/ aveia+pastagem/soja/formação da pastagem/ pastagem. A braquária em consórcio com o milho tem a função de melhorar a cobertura do solo com sua palhada após a colheita do milho. A carga animal nas áreas de pastagem é de 3,5 cab ha-1.

Foram realizadas duas amostragens, sendo a primeira na época seca (maio de 2009) onde 
as culturas que se encontravam na área eram pasto, cujo talhão avaliado possuía 43,04 ha; milho+braquiária que se encontravam no estádio vegetativo em talhão de 51 ha; e algodão recém colhido em talhão de 57,29 ha, ambos conduzidos no sistema integração lavoura-pecuária, além de uma área de Cerradão, que foi utilizada como referência. A segunda amostragem foi realizada na época chuvosa (março de 2010) e as culturas presentes no momento da amostragem eram milho, que estava consorciado com braquiária e algodão, ambos em estádio vegetativo e soja recém colhida. As áreas nas duas épocas de avaliação foram: Cerradão, pasto/milho, milho/algodão e algodão/ soja. A área de pasto/milho corresponde à avaliação de pasto na época seca e milho na época chuvosa; a área de milho/algodão corresponde à avaliação de milho+braquiária na época seca e algodão na época chuvosa, o solo nessas áreas foi identificado como LATOSSOLO VERMELHO. A área de algodão/ soja encontrava-se com algodão na época seca e soja na época chuvosa, nessa área o solo foi classificado como LATOSSOLO VERMELHO-AMARELO.

Para a coleta das amostras para avaliação dos agregados em cada uma das áreas foram abertas seis trincheiras com dimensões aproximadas de $25 \mathrm{~cm} \mathrm{x}$ $20 \mathrm{~cm}$. As amostras foram coletadas na camada de 0-10 cm de profundidade com auxílio de uma pá quadrada.

Após a coleta, as amostras foram submetidas a peneiramento no campo, sendo utilizado um conjunto de peneiras de 9,7 e 8,0 mm, os agregados identificados foram aqueles com tamanho entre 8,0 e $9,7 \mathrm{~mm}$, os agregados maiores que $9,7 \mathrm{~mm}$ e menores que $8,0 \mathrm{~mm}$ foram descartados. Os agregados obtidos foram levados para laboratório e observados sob lupa e separados à mão em frações morfológicas, identificando-os em classes de acordo com as definições descritas em Bullock et al. (1985).

A separação dos agregados foi feita através de padrões morfológicos, sendo estabelecidos três tipos de agregados (Figura 1): (a) fisiogênicos - definidos por apresentarem formas angulares; (b) biogênicos - aqueles onde foi possível a visualização de formas arredondadas, providas pelo trato intestinal dos indivíduos da macrofauna do solo, principalmente Oligochaeta e/ou aqueles em que era possível visualizar a atividade de raízes, e (c) intermediários - agregados que possuíam forma indefinida, podendo ser aqueles biogênicos que perderam a forma arredondada devido ao envelhecimento ou fisiogênico que possuía uma solda com um pequeno coprólito

Para determinação da contribuição relativa em massa foram pesados $100 \mathrm{~g}$ de agregados de cada repetição e área. A massa de agregados foi identificada sob lupa binocular e assim quantificada a fração de agregados fisiogênicos, intermediários e biogênicos contidos na massa inicial. Após a separação os agregados foram submetidos à análise de estabilidade em água (EMBRAPA, 1997) e determinação dos teores de $\mathrm{Ca}, \mathrm{Mg}, \mathrm{K}, \mathrm{Na}$, $\mathrm{Al}, \mathrm{H}+\mathrm{Al}, \mathrm{P}$ e pH em água (EMBRAPA, 1997) e carbono orgânico total (YEOMANS; BREMNER, 1988).

Foi avaliada a densidade das Oligochaetas (minhocas), Isoptera (cupins) e Larvas de Coleoptera (larvas de besouros) a partir do método de monólitos de solo recomendado pelo Programa Tropical Soil Biology and Fertility (TSBF) descrito por Anderson e Ingram (1993), na profundidade de 0-10 cm. Na mesma profundidade foi coletada uma amostra de terra para realização da análise granulométrica do solo segundo Day (1965), determinação dos teores de $\mathrm{Ca}, \mathrm{Mg}, \mathrm{K}, \mathrm{Na}, \mathrm{Al}, \mathrm{H}+\mathrm{Al}, \mathrm{P}$ e pH em água (EMBRAPA, 1997), fracionamento granulométrico da matéria orgânica do solo (CAMBARDELLA; ELLIOT, 1992), matéria orgânica leve em água (ANDERSON; INGRAM, 1989) e frações oxidáveis do carbono orgânico total (CHAN; BOWMAN; OATES, 2001). 
Figura 1. Exemplo da separação dos diferentes tipos de agregados da fração 9,7-8,0 $\mathrm{mm}$. (A) agregado fisiogênico, (B) agregado intermediário e (C) agregado biogênico.
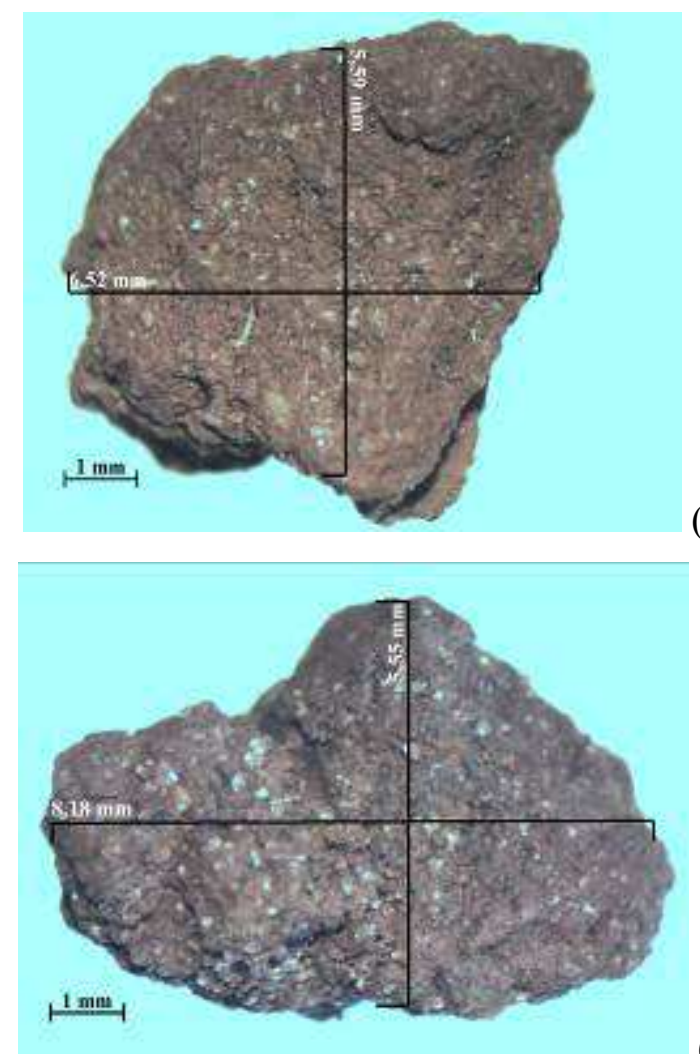

(A)

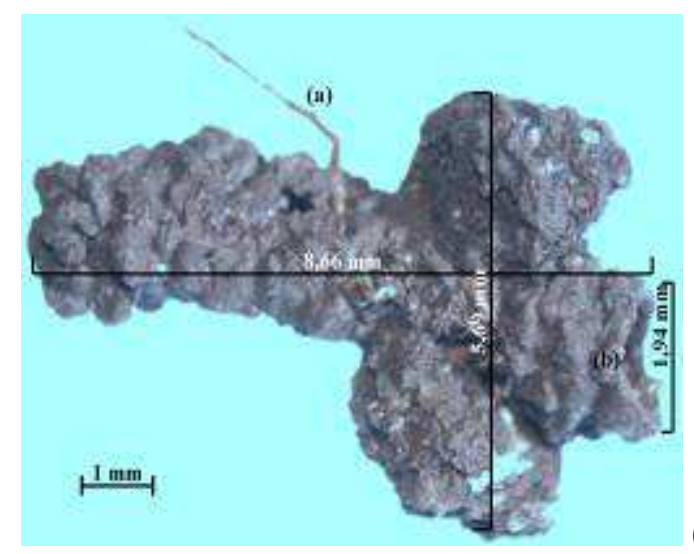

(B)

(C)

Fonte: Elaboração dos autores.

Para os dados obtidos, foi feita uma avaliação da normalidade dos dados (LILLIEFORS) e homogeneidade das variâncias dos erros (COCHRAN; BARTTLET). Quando os dados atenderam as pressuposições da análise paramétrica, os valores médios comparados entre si pelo teste $\mathrm{t}$ de Bonferroni a 5\% de probabilidade, quando não atenderam as pressuposições os valores foram comparados pelo teste não paramétrico de Kruskal Wallis.

Com o objetivo de se verificar a ocorrência de um gradiente de qualidade do solo, foi realizada uma análise multivariada, denominada de Análise de Redundância (Redundancy Analysis ou RDA) com o auxílio do programa CANOCO 4.5. O pressuposto da RDA é que as características dos agregados são resultado de interferências pontuais, como a ação da biota local (fauna) e de circunstâncias do manejo e da época, o que configura um outro nível de influência, de maior escala. Desta forma, consideraram-se como variáveis dependentes as características dos agregados e como independentes as variáveis da fauna, do manejo e as épocas. Foi realizada uma análise prévia para verificar a existência de colinearidade e retirar as variáveis colineares, melhorando o percentual de explicação e aumentando a significância dos eixos canônicos. Dessa forma, foram mantidas para os agregados fisiogênicos, intermediários e biogênicos as variáveis relativas à: quantidade, diâmetro médio ponderado (DMP), Ca, P, pH, C e valor V. Para a fauna foram utilizadas as densidades dos seguintes grupos taxonômicos: Isoptera, Larvas de Coleoptera, Oligochaeta e Riqueza Média que representa o número médio de grupos da fauna coletados por amostra, em cada área, além de todos os manejos e épocas.

\section{Resultados e Discussão}

$\mathrm{Na}$ época seca foram verificadas diferenças entre as três vias de formação de agregados, com maiores valores de porcentagem de composição para os intermediários (Tabela 1), seguidos pelos fisiogênicos e os menores valores para os biogênicos. Já na época chuvosa, os valores percentuais de agregados somente diferiram na área de algodão/soja, o que pode ser decorrente 
do menor teor de argila observado nessa área, que caracteriza área como textura média, favorecendo o aumento da decomposição de matéria orgânica (PILON; MIELNICZUK; MARTIN NETO, 2002). As demais áreas apresentaram teor de argila que as enquadra como de textura argilosa. O carbono apresentou correlação positiva para o processo de formação de agregados biogênicos na época seca e chuvosa com correlações de $81 \%$ e $60 \%$ respectivamente (Tabelas 2 e 3), o que está de acordo com Brussaard et al. (2007) que dizem que agregados biogênicos são sítios importantes para a estabilização da matéria orgânica

Quanto aos valores de DMP, esses não diferiram quanto às vias de formação. Verificaram-se elevados valores desses índices, próximos a 5,000 mm, indicando uma elevada estabilidade estrutural, o que pode ser atribuído à composição de oxídica dos Latossolos. De acordo com a análise de correlação (Tabelas 2 e 3), o carbono não apresenta um padrão de correlação com o DMP, que foi o atributo utilizado para avaliar a estabilidade dos agregados, ora sendo observada correlação positiva, ora negativa. Como o DMP apresenta valores próximos ao maior intervalo de classe, independente da correlação com o carbono, conclui-se que outras características intrínsecas do solo é que estão promovendo a elevada estabilidade dos agregados. De acordo com Resende et al. (1995), Latossolos apresentam naturalmente elevada estabilidade estrutural, devido à atuação de óxidos e matéria orgânica. Associado às características próprias dessa ordem de solos, o sistema conservacionista utilizado propicia condições de elevada estabilidade dos agregados, devido ao não revolvimento do solo e cobertura constante.
$\mathrm{Na}$ Tabela 4 são apresentados os valores percentuais dos agregados formados pelas diferentes vias, DMP e diâmetro médio geométrico (DMG), e as comparações entre áreas e épocas de coleta. Não foram observadas diferenças entre as épocas de avaliação, pois de acordo com a Tabela 5 não houve diferença de densidade de indivíduos da macrofauna entre as épocas avaliadas, sendo esses, os agentes de formação dos agregados biogênicos. As áreas de produção não apresentaram diferença quanto ao DMP e DMG em relação à vegetação nativa devido o ILP proporcionar conservação das propriedades físicas do solo, o que corrobora com Calegari et al. (2006) que realizaram estudo com diferentes sistemas de manejo, sendo esses: plantio direto e convencional em área de Latossolo no Paraná, não constataram diferenças significativas entre vegetação nativa e o sistema de plantio direto nos valores de DMP e DMG. Os autores atribuem esse padrão aos efeitos benéficos do acúmulo de resíduos orgânicos na superfície do solo no sistema de plantio direto, onde, além do solo não ser revolvido, práticas como rotação de culturas e uso adequado de plantas de cobertura, favorecem a melhoria da agregação. Segundo Bronick e Lal (2005), a agregação do solo pode ser significativamente modificada através de práticas de manejo e alterações no meio ambiente, assim, práticas que diminuem a perturbação do solo aumentam a agregação e o desenvolvimento estrutural. 


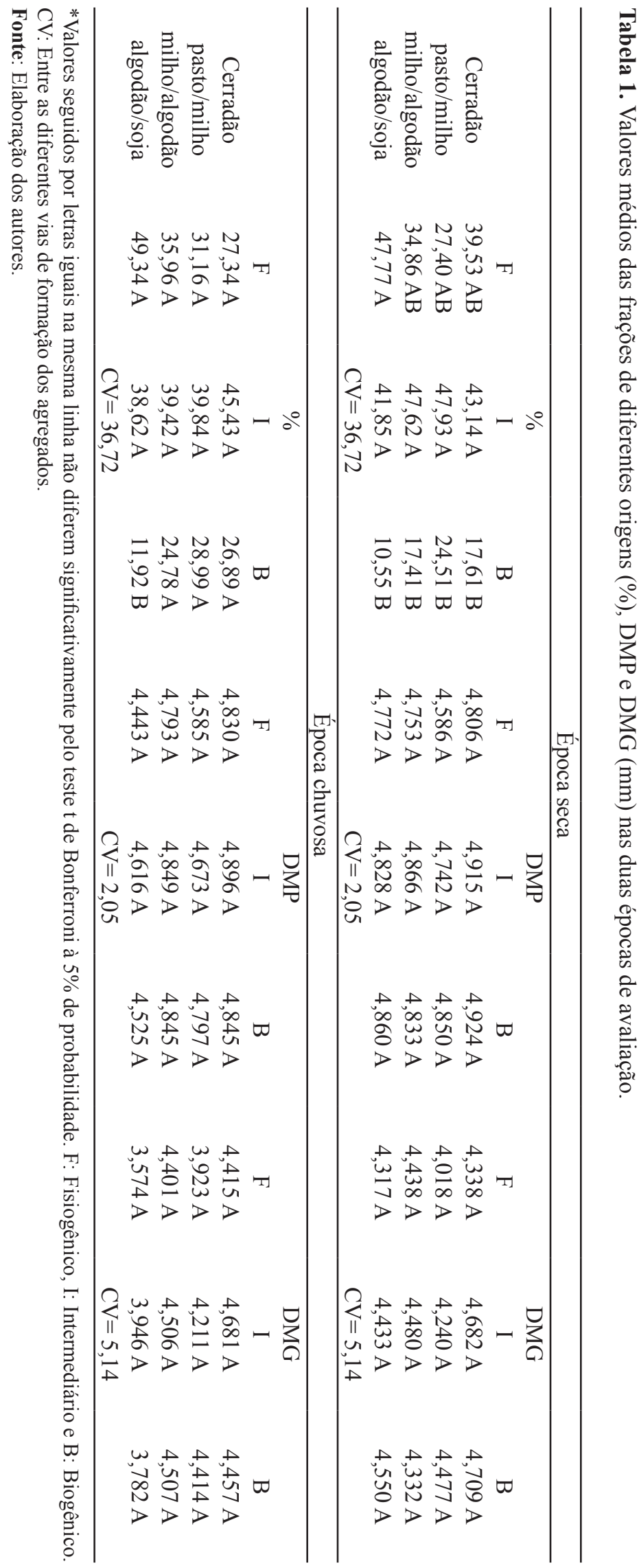


Tabela 2 Matriz de correlação entre os principais parâmetros ultilizados na época seca de avaliação.

\begin{tabular}{|c|c|c|c|c|}
\hline & Fisiogênico & & & \\
\hline & Quantidade & Carbono & Oligochaeta & DMP \\
\hline Quantidade & 1 & $-0,4884$ & $-0,0996$ & $0,7895^{* *}$ \\
\hline Carbono & & 1 & $-0,4115$ & $-0,1125$ \\
\hline Oligochaeta & & & 1 & 0,2017 \\
\hline \multirow[t]{3}{*}{ DMP } & & & & 1 \\
\hline & & Intermedi & & \\
\hline & Quantidade & Carbono & Oligochaeta & DMP \\
\hline Quantidade & 1 & $-0,5609$ & 0,4626 & $-0,4687$ \\
\hline Carbono & & 1 & $-0,1951$ & $0,8804 * *$ \\
\hline Oligochaeta & & & 1 & 0,2592 \\
\hline \multirow[t]{3}{*}{ DMP } & & & & 1 \\
\hline & & Biogênico & & \\
\hline & Quantidade & Carbono & Oligochaeta & DMP \\
\hline Quantidade & 1 & $0,8143^{*}$ & $-0,1191$ & $-0,0958$ \\
\hline Carbono & & 1 & $-0,6296$ & 0,4225 \\
\hline Oligochaeta & & & 1 & $-0,5967$ \\
\hline DMP & & & & 1 \\
\hline
\end{tabular}

* e ** : significativos a 10 e $5 \%$

Fonte: Elaboração dos autores.

Tabela 3. Matriz de correlação entre os principais parâmetros ultilizados na época chuvosa de avaliação.

\begin{tabular}{|c|c|c|c|c|}
\hline & Fisiogênico & & & \\
\hline & Quantidade & Carbono & Oligochaeta & DMP \\
\hline Quantidade & 1 & $-0,8849 * *$ & $-0,3454$ & $-0,7657$ \\
\hline Carbono & & 1 & 0,5354 & 0,47680 \\
\hline Oligochaeta & & & 1 & $-0,3293$ \\
\hline DMP & & & & 1 \\
\hline & Intermediáric & & & \\
\hline & Quantidade & Carbono & Oligochaeta & DMP \\
\hline Quantidade & 1 & 0,6739 & $-0,0462$ & 0,7193 \\
\hline Carbono & & 1 & 0,6986 & 0,2496 \\
\hline Oligochaeta & & & 1 & $-0,4398$ \\
\hline DMP & & & & 1 \\
\hline & Biogênico & & & \\
\hline & Quantidade & Carbono & Oligochaeta & DMP \\
\hline Quantidade & 1 & 0,6019 & 0,4487 & $0,9354 * *$ \\
\hline Carbono & & 1 & 0,1896 & 0,5750 \\
\hline Oligochaeta & & & 1 & 0,1044 \\
\hline DMP & & & & 1 \\
\hline
\end{tabular}

$*$ e** : significativos a 10 e $5 \%$

Fonte: Elaboração dos autores. 
Tabela 4. Valores médios do peso das frações de diferentes origens (\%), DMP e DMG (mm) nas duas épocas de avaliação.

\begin{tabular}{|c|c|c|c|c|c|c|}
\hline & \multicolumn{6}{|c|}{ Fisiogênico } \\
\hline & \multicolumn{2}{|c|}{$\%$} & \multicolumn{2}{|c|}{ DMP } & \multicolumn{2}{|c|}{ DMG } \\
\hline & $\mathrm{S}$ & $\mathrm{C}$ & $\mathrm{S}$ & $\mathrm{C}$ & $\mathrm{S}$ & $\mathrm{C}$ \\
\hline Cerradão & $39,53 \mathrm{Aa}$ & $27,34 \mathrm{Aa}$ & $4,806 \mathrm{Aa}$ & $4,830 \mathrm{Aa}$ & $4,338 \mathrm{Aa}$ & $4,415 \mathrm{Aa}$ \\
\hline pasto/milho & $27,40 \mathrm{Aa}$ & $31,16 \mathrm{Aa}$ & $4,586 \mathrm{Aa}$ & $4,585 \mathrm{Aa}$ & $4,018 \mathrm{Aa}$ & $3,923 \mathrm{Aa}$ \\
\hline milho/algodão & $34,86 \mathrm{Aa}$ & $35,96 \mathrm{Aa}$ & $4,753 \mathrm{Aa}$ & 4,793 Aa & $4,438 \mathrm{Aa}$ & $4,401 \mathrm{Aa}$ \\
\hline \multirow[t]{5}{*}{ algodão/soja } & $47,77 \mathrm{Aa}$ & $49,34 \mathrm{Aa}$ & $4,772 \mathrm{Aa}$ & 4,443 Aa & $4,317 \mathrm{Aa}$ & $3,574 \mathrm{Aa}$ \\
\hline & \multicolumn{2}{|c|}{$\mathrm{CV}(1)=0,97$} & \multicolumn{2}{|c|}{$\mathrm{CV}(1)=3,67$} & \multicolumn{2}{|c|}{$C V(1)=8,39$} \\
\hline & \multicolumn{2}{|c|}{$\mathrm{CV}(2)=33,10$} & \multicolumn{2}{|c|}{$\mathrm{CV}(2)=4,04$} & \multicolumn{2}{|c|}{$\mathrm{CV}(2)=9,39$} \\
\hline & \multicolumn{6}{|c|}{ Intermediário } \\
\hline & \multicolumn{2}{|c|}{$\%$} & \multicolumn{2}{|c|}{ DMP } & \multicolumn{2}{|c|}{$\overline{D M G}$} \\
\hline Cerradão & $43,14 \mathrm{Aa}$ & $45,43 \mathrm{Aa}$ & $4,915 \mathrm{Aa}$ & 4,896 Aa & $4,682 \mathrm{Aa}$ & $4,681 \mathrm{Aa}$ \\
\hline pasto/milho & 47,93 Aa & $39,84 \mathrm{Aa}$ & $4,742 \mathrm{Aa}$ & $4,673 \mathrm{Aa}$ & $4,240 \mathrm{Aa}$ & $4,211 \mathrm{Aa}$ \\
\hline milho/algodão & $47,62 \mathrm{Aa}$ & $39,42 \mathrm{Aa}$ & $4,866 \mathrm{Aa}$ & 4,849 Aa & $4,480 \mathrm{Aa}$ & $4,506 \mathrm{Aa}$ \\
\hline \multirow[t]{5}{*}{ algodão/soja } & $41,85 \mathrm{Aa}$ & $38,62 \mathrm{Aa}$ & $4,828 \mathrm{Aa}$ & 4,616 Aa & 4,433 Aa & $3,946 \mathrm{Aa}$ \\
\hline & \multicolumn{2}{|c|}{$\mathrm{CV}(1)=0,97$} & \multicolumn{2}{|c|}{$\mathrm{CV}(1)=3,67$} & \multicolumn{2}{|c|}{$\mathrm{CV}(1)=8,39$} \\
\hline & \multicolumn{2}{|c|}{$\mathrm{CV}(2)=33,10$} & $\mathrm{CV}$ & 4,04 & \multicolumn{2}{|c|}{$\operatorname{CV}(2)=9,39$} \\
\hline & & & \multicolumn{2}{|c|}{ Biogênico } & & \\
\hline & \multicolumn{2}{|c|}{$\%$} & \multicolumn{2}{|c|}{ DMP } & \multicolumn{2}{|c|}{$\mathrm{DMG}$} \\
\hline Cerradão & $17,61 \mathrm{Aa}$ & $26,89 \mathrm{Aa}$ & 4,924 Aa & $4,845 \mathrm{Aa}$ & 4,709 $\mathrm{Aa}$ & $4,457 \mathrm{Aa}$ \\
\hline pasto/milho & $24,51 \mathrm{Aa}$ & $28,99 \mathrm{Aa}$ & $4,850 \mathrm{Aa}$ & 4,797 $\mathrm{ABa}$ & $4,477 \mathrm{Aa}$ & $4,414 \mathrm{Aa}$ \\
\hline milho/algodão & $17,41 \mathrm{Aa}$ & $24,78 \mathrm{Aa}$ & $4,833 \mathrm{Aa}$ & $4,845 \mathrm{Aa}$ & 4,332 Aa & $4,507 \mathrm{Aa}$ \\
\hline \multirow[t]{3}{*}{ algodão/soja } & $10,55 \mathrm{Aa}$ & $11,92 \mathrm{Aa}$ & $4,860 \mathrm{Aa}$ & $4,525 \mathrm{Ba}$ & $4,550 \mathrm{Aa}$ & $3,782 \mathrm{Ab}$ \\
\hline & \multicolumn{2}{|c|}{$\mathrm{CV}(1)=0,97$} & \multicolumn{2}{|c|}{$\mathrm{CV}(1)=3,67$} & \multicolumn{2}{|c|}{$\mathrm{CV}(1)=8,39$} \\
\hline & \multicolumn{2}{|c|}{$\mathrm{CV}(2)=33,10$} & \multicolumn{2}{|c|}{$\mathrm{CV}(2)=4,04$} & \multicolumn{2}{|c|}{$\mathrm{CV}(2)=9,39$} \\
\hline
\end{tabular}

Valores seguidos por letras maiúsculas iguais na coluna e minúsculas iguais na linha não diferem significativamente pelo teste $t$ de Bonferroni a 5\%. S: época seca e C: época chuvosa. $\mathrm{CV}(1)=$ coeficiente de variação entre áreas, $\mathrm{CV}(2)=$ coeficiente de variação entre épocas de avaliação.

Fonte: Elaboração dos autores.

Tabela 5. Densidade total da macrofauna edáfica $\left(\right.$ ind $\mathrm{m}^{-2}$ ).

\begin{tabular}{ccc}
\hline & Época seca & Época chuvosa \\
Áreas & Ind m $^{2}$ & Ind $^{2}$ \\
Cerradão & $864 \pm 300 \mathrm{Aa}$ & $2013 \pm 662 \mathrm{Aa}$ \\
pasto/milho & $400 \pm 284 \mathrm{~A} \mathrm{a}$ & $453 \pm 106 \mathrm{Aa}$ \\
milho/algodão & $363 \pm 137 \mathrm{~A} \mathrm{a}$ & $216 \pm 76 \mathrm{Aa}$ \\
algodão/soja & $133 \pm 60 \mathrm{~A} \mathrm{a}$ & $136 \pm 45 \mathrm{Aa}$ \\
\hline
\end{tabular}

Valores seguidos por letras maiúsculas iguais na coluna e minúsculas iguais na linha não diferem significativamente pelo teste Kruskal Wallis.

Fonte: Elaboração dos autores. 
No diagrama de ordenação resultante da análise de redundância (Figura 2), observa-se que existe uma relação estreita entre a área de Cerradão e as diferentes formas de Carbono (COp, COam, F2), assim como com o carbono dos diferentes tipos de agregados. As áreas de produção com características semelhantes como milho/algodão e pasto/milho, se mostram agrupadas por esta análise. Essas áreas apresentam gramíneas como culturas recentes, associadas a essas está à fração F1 da matéria orgânica que é aquela que se apresenta em estádio inicial de decomposição, sendo, portanto, fonte de alimento para indivíduos da macrofauna edáfica como os Oligochaeta. Resultado disso é que de acordo com a análise, Oligochaeta apresenta forte relação com a quantidade de agregados de origem biogênica. Segundo Jongmans et al. (2003), os Oligochaeta são importantes engenheiros do ecossistema e atuam na formação de agregados ricos em matéria orgânica.

Figura 2. Análise de Redundância (RDA) das características químicas dos diferentes tipos de agregados em função da macrofauna edáfica, variáveis químicas e manejo do solo. Sendo cot: carbono orgânico total, cop: carbono orgânico particulado, coam: carbono orgânico associado aos minerais, mol: matéria orgânica leve em água, F1, F2, F3 e F4: frações oxidáveis da matéria orgânica, carb: carbono, Isop: Isoptera, Lcoleop: Larvas de Coleoptera, Oligoch: Oligochaeta e RiqMed: Riqueza média. As variáveis a seguir, são precedidas de $\mathrm{f}$ : fisiogênico, i: intermediário e b: biogênico, são elas, dmp: diâmetro médio ponderado, dmg: diâmetro médio geométrico, qtde: composição, $\mathrm{P}, \mathrm{V}, \mathrm{pH}, \mathrm{Ca}$.

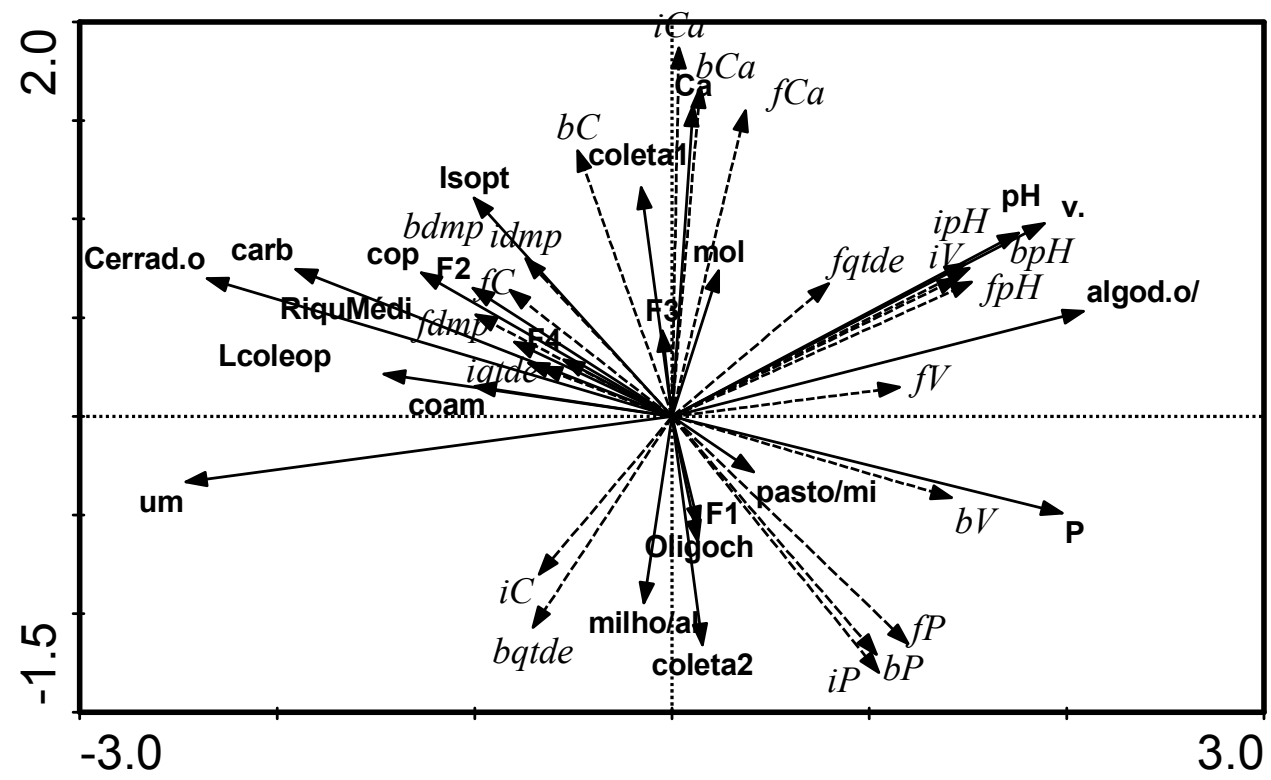

Fonte: Elaboração dos autores.

Em contrapartida a área de algodão/soja está associada a uma maior quantidade de agregados de origem fisiogênica, provavelmente por possuir textura média, enquanto as demais áreas apresentam textura argilosa. Portanto, quanto maior o teor de areia, menor a proteção da matéria orgânica e consequentemente menor disponibilidade de alimento para a macrofauna edáfica. Segundo
Pilon, Mielniczuk e Martin Neto (2002) em um solo arenoso, o arranjo das partículas primárias resulta em uma distribuição de poros de maior tamanho, o que facilita o acesso microbiano à matéria orgânica. De acordo com Dias et al. (2012) quanto maior o teor de areia, menor a possibilidade de se encontrar Isoptera (cupins) e segundo Freitas (2007), o fato de o solo ser mais arenoso dificulta a formação 
das galerias pelos Oligochaetas (minhocas), pois à medida que aumenta o teor de areia, aumenta a abrasividade do solo, ou seja, o atrito entre as partículas e o corpo das minhocas, inviabilizando a colonização desses solos (LEE, 1985).

A ordem Isoptera está associada à área de Cerradão, mas também, ao atributo DMP dos diferentes tipos de agregados, segundo GarnierSilllam, Toutain e Renoux (1988) os Isoptera por meio de suas ações mecânicas modificam a estabilidade dos agregados. Dessa forma é possível observar como o ambiente influencia no comportamento da macrofauna e a resposta que ela fornece modificando o ambiente habitado.

O conjunto de todas as variáveis, explica $92,3 \%$ de toda a variabilidade dos diferentes tipos de agregados analisados (Figura 2). Na Tabela 6, é apresentado o teste de permutação que demonstra relações significativas entre as variáveis dependentes (características dos agregados) e as variáveis independentes (características do solo, manejo e épocas). Ou seja, os fatores ambientais testados determinam de maneira significativa as características dos agregados. No entanto, pelo procedimento de partição da variabilidade, é possível determinar o grau de influência de cada grupo dos fatores ambientais. As variáveis independentes que mais influenciaram a variabilidade dos diferentes tipos de agregados foram umidade, a cobertura vegetal (Cerradão e Algodão/Soja), V\% do solo, teores de $\mathrm{P}$ e carbono do solo. A umidade é uma variável muito importante para que ocorra atividade biológica, sendo ao mesmo tempo resultado de outros fatores ambientais, tais como a cobertura vegetal. Segundo Lavelle (1983), a umidade é a variável ambiental mais importante na distribuição e abundância de Oligochaeta em solos tropicais.

A área de Cerradão foi importante na distinção das características dos diferentes tipos de agregados pelo fato de ser um ambiente nativo, assim, as características ali manifestadas referese às condições naturais onde não se verifica ação antrópica ou se elas são realizadas promovem um baixo impacto. A área de algodão/soja foi importante na variabilidade das características, devido ter sido a que apresentou menores teores de carbono em relação às demais. $\mathrm{O} \mathrm{V} \%$ assim como os teores de $\mathrm{P}$ refletem as características químicas dos agregados formados, já o carbono tem influência na variabilidade dos diferentes tipos de agregados devido apresentar correlação positiva e estreita na formação de agregados biogênicos.

Com base nas características químicas analisadas, verifica-se que os agregados apresentam características químicas distintas do solo que os originaram (Figura 3). Diversos são os fatores que interferem na agregação do solo, propiciando ao agregado formado características químicas diferentes do solo de origem, principalmente no que se refere à matéria orgânica e as características que a envolvem. Os três tipos de agregados apresentaram um padrão de distinção; agregados fisiogênicos são mais próximos dos agregados intermediários, estes por sua vez se distanciam dos agregados biogênicos. Este padrão de separação reflete o quanto a origem do agregado pode influenciar em diferentes características químicas. 
Tabela 6. Teste de permutação das variáveis de solo, em que apresentaram explicação total de $92,3 \%$ para a variabilidade de características de agregados biogênicos, fisiogênicos e intermediários.

\begin{tabular}{ccc}
\hline Variável & Explicação isoladamente para cada variável & significância \\
\hline Umidade & 0,257 & 0,002 \\
Cerradão & 0,255 & 0,002 \\
Algodão/Soja & 0,212 & 0,002 \\
V\% do solo & 0,196 & 0,002 \\
P do solo & 0,184 & 0,002 \\
Carbono do solo & 0,174 & 0,002 \\
pH do solo & 0,161 & 0,002 \\
COp do solo & 0,123 & 0,010 \\
Ca do solo & 0,120 & 0,012 \\
Larvas de Coleoptera & 0,099 & 0,022 \\
F2 do solo & 0,099 & 0,030 \\
Isoptera & 0,098 & 0,046 \\
Coleta 1 & 0,087 & 0,042 \\
Coleta 2 & 0,087 & 0,044 \\
Riqueza média da fauna & 0,072 & 0,104 \\
Pasto/milho & 0,070 & 0,092 \\
Milho/Algodão & 0,070 & 0,104 \\
Coam do solo & 0,070 & 0,0840 \\
F1 do solo & 0,050 & 0,276 \\
MOL do solo & 0,048 & 0,328 \\
Oligochaeta & 0,032 & 0,630 \\
F4 do solo & 0,032 & 0,684 \\
F3 do solo & 0,028 & 0,641 \\
\hline
\end{tabular}

COp do solo: carbono orgânico particulado; COam: carbono orgânico associado aos minerais; F1, F2, F3 e F4: frações oxidáveis do carbono.

Fonte: Elaboração dos autores.

Figura 3. Dendrograma resultante da análise de agrupamento, evidenciando as distâncias entre as características químicas $(\mathrm{H}+\mathrm{Al}, \mathrm{Ca}, \mathrm{Mg}, \mathrm{P}, \mathrm{Na}, \mathrm{K}, \mathrm{pH}, \mathrm{C}, \mathrm{S}, \mathrm{T}$ e V) dos materiais testados (solo e agregados dos tipos fisiogênico, intermediário e biogênico). Distância Eucliana e modo de ligação completa.

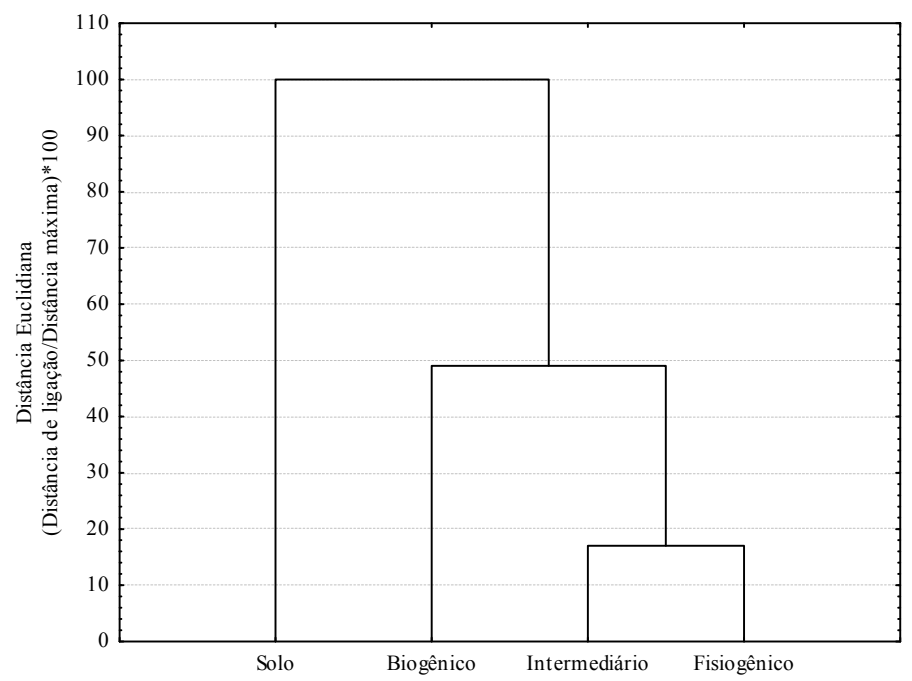

Fonte: Elaboração dos autores. 


\section{Conclusões}

Em todas as áreas estudadas, na época seca, foram quantificados os maiores valores de agregados intermediários, enquanto na época chuvosa, de maneira geral, não foram observadas diferenças dos agregados formados pelas diferentes vias nas áreas, exceto algodão/soja;

Os agregados biogênicos foram encontrados em menor quantidade e apresentaram correlação positiva com teores de carbono;

Isoptera influencia na estabilidade de agregados e Oligochaeta na quantidade de agregados biogênicos formados;

Os diferentes tipos de agregados formados, além de apresentarem características morfológicas diferentes, também apresentam diferenças quanto às características químicas.

\section{Referências}

ANDERSON, J. M.; INGRAM, J. S. I. Tropical soil biology and fertility: a handbook of methods. Wallingford, CAB International, 1989. $171 \mathrm{p}$.

Tropical soil biological and fertility: a handbook of methods. 2. ed. Wallingford: CAB International, 1993. $221 \mathrm{p}$.

BALBINO, L. C.; BRUAND, A.; BROSSARD, M.; GRIMALDI, M.; HAJNOS, M.; GUIMARÃES, M. F. Changes in porosity and microaggregation in clayey Ferralsols of the Brazilian Cerrado on clearing for pasture. European Journal of Soil Science, Oxford, v 53, n. 53, p. 219-230, 2002.

BASTOS, R. S.; MENDONÇA, E. S.; ALVAREZ, V. H.; CORRÊA, M. M.; COSTA, L. M. Formação e estabilização de agregados do solo influenciados por ciclos de umedecimento e secagem após adição de compostos orgânicos com diferentes características hidrofóbicas. Revista Brasileira de Ciência do Solo, Viçosa, MG, v. 29, n. 1, p. 21-31, 2005.

BRONICK, C. J.; LAL, R. Soil structure and management: a review. Geoderma, Amsterdam, v. 124, n. 1-2, p. 3-22, 2005.

BRUSSAARD, L.; PULLEMAN, M. M.; OUE'DRAOGO, E.; MANDO, A.; SIX, J. Soil fauna and soil function in the fabric of the food web. Pedobiologia, Jena, v. 50, n. 6, p. 447-462, 2007.
BULLOCK, P.; FEDEROFF, N.; JONGERIUS, A.; STOOPS, G.; TURSINA, T. Handbook for soil thin section description. Albrighton, England: Waine Research Publications, 1985. $152 \mathrm{p}$.

CALEGARI, A.; CASTRO FILHO, C.; TAVARES FILHO, J.; RALISCH, R.; GUIMARÃES, M. F. Melhoria da agregação do solo através do sistema plantio direto. Revista Semina: Ciências Agrárias, Londrina, v. 27, n. 2, p.148-158, abr./jun. 2006.

CAMBARDELla, C. A.; ElLIOTT, E. T. Particulate soil organic matter changes across a grassland cultivation sequence. Soil Science Society of American Journal, Madison, v. 56, n. 3, p. 777-783, 1992.

CARVALHO, A. F. Emprego da agitação horizontal na avaliação da estabilidade de agregados de cinco solos da região sudeste. 1991. Dissertação (Mestrado em Agronomia/Solos e Nutrição de Plantas) - Universidade Federal de Viçosa, Viçosa, MG.

CHAN, K. Y.; BOWMAN, A.; OATES, A. Oxidizible organic carbon fractions and soil quality changes in an Paleustalf under different pasture leys. Soil Science, Madison, v. 166, n. 1, p. 61-67, 2001.

DAY, P. R. Particle fractionation and particle-size analysis. In: BLACK, C. A. (Ed.). Methods of soil analysis. Madison: American Society of Agronomy, 1965. v. 1, p. 545-556.

DECAËNS, T.; GALVIS, J. H.; AMÉZQUITA, E. Propriétés des structures produites par les ingénieurs écologiques à la surface du sol d'une savane colombienne. Comptes Rendus de l'Académie des Sciences - Series III - Sciences de la Vie., Paris, v. 324, n. 5, maio, p. 465-478, 2001.

DIAS, N. P.; MEDEIROS, L. R.; PAZINI, J. P.; SIKVA, F. F. Distribuição espacial de Procornitermes sp. (Isoptera: Termitidae) em função das propriedades físicas do solo em área de pastagem no município de João Borba, Rio Grande do Sul. Revista Brasileira de Agroecologia, Bahia, v. 7, n. 2, p. 104-111, 2012.

EMPRESA BRASILEIRA DE PESQUISA AGROPECUÁRIA - EMBRAPA. Centro Nacional de Pesquisa de Solos. Manual de métodos de análise de solo. 2. ed. Rio de Janeiro: Embrapa, 1997. 212 p.

FREITAS, M. P. Flutuação populacional de Oligochaeta edáficos em hortas sob sistemas convencional e orgânico no municipio de Canoinhas/SC. 2007. Dissertação (Mestrado em Ciência do Solo) - Universidade Federal do Paraná, Curitiba.

GARNIER-SILLAM, E.; TOUTAIN, F.; RENOUX, J. Comparaison de l'influence de deux termitières (humivore et champignonniste) sur la stabilité structurale des sols forestiers tropicaux. Pedobiologia, Jena, v. 32, n. 1-2, p. 89-97, 1988. 
INSTITUTO BRASILEIRO DE GEOGRAFIA E ESTATÍSTICA - IBGE. Indicadores de desenvolvimento sustentável. Estudos e pesquisas. Rio de Janeiro: IBGE, 2010. 443 p. (Informação geográfica, 7).

INGHAN, E. R. The soil food web. In: SOIL AND WATER CONSERVATION SOCIETY. Soil biology primer. Iowa, 2000. cap. 1. Disponível em: $<$ http://www. soils.usda.gov/sqi/concepts/soil_biology/soil_food_web. html>. Acesso em: 02 nov. 2012.

JONGMANS, A. G.; PULLEMAN, M. M.; BALABANE, M.; VAN OORT, F.; MARINISSEN, J. C. Y. Soil structure and characteristics of organic matter in two orchards differing in earthworm activity. Applied Soil Ecology, Amsterdam, v. 24, n.3, p. 219-232, 2003.

JONGMANS, A. G.; PULLEMAN, M. M.; MARINISSEN, J. C. Y.; Soil structure and earthworm activity in a marine silt loam under pasture versus arable land. Biology and Fertility of Soils, Germany, v. 33, n. 4, p. 279-285, 2001.

LAVELLE, P. The soil fauna of tropical savannas. The community structure. In: BOURLIÈRE, F. (Ed.). Tropical savannas. Amsterdam: E. S. P. C., 1983. cap. 21, p. 477-484.

LEE, K. E. Earthworms: their ecology and relationships with soil and land use. New York: Academic Press, 1985.

MACEDO, M. C. M. Integração lavoura e pecuária: alternativa para sustentabilidade da produção animal. In: SIMPÓSIO SOBRE MANEJO DA PASTAGEM, 2001, Piracicaba. Anais... Piracicaba: FEALQ, 2001. p. 257283.

MARCHÃO, R. L. Integração lavoura-pecuária num latossolo do cerrado: impacto na física, matéria orgânica e macrofauna. 2007. Tese (Doutorado em Agronomia/ Solo e Água) - Universidade Federal de Goiás, Goiânia.

MELLO, N. A.; CÉCILLON, L.; BRUN, J. J. Formação e propriedades de Macroagregados de um solo alpino sob três tipos de vegetação nativa. In: REUNIÃO BRASILEIRA DE MANEJO E CONSERVAÇÃO DO SOLO E DA ÁGUA: NO CONTEXTO DAS MUDANÇAS AMBIENTAIS, 27., 2008. Rio de Janeiro. Anais... Rio de Janeiro: SBS, 2008. CD-ROM.

PILON, C. N.; MIELNICZUK, J.; MARTIN NETO, L. Dinâmica da matéria orgânica no ambiente. Pelotas: Embrapa Clima Temperado, 2002. 41 p. (Documentos, 105).

PULLEMAN, M. M.; MARINISSEN, J. C. Y. Physical protection of mineralizable $\mathrm{C}$ in aggregates from longterm pasture and arable soil. Geoderma, Amsterdam, v. 120, n. 3-4, p. 273-282, 2004.
PULLEMAN, M. M.; SIX, J.; MARINISSEN, J. C. Y.; JONGMANS, A. G.; Earthworms and management affect organic matter incorporation and microaggregate formation in agricultural soils. Applied Soil Ecology, Amsterdam, v. 29, n. 1, p. 1-15, 2005.

REATTO, A.; BRUAND, A.; MARTINS, E. S.; MULLER, F.; SILVA, E. M.; CARVALHO JÚNIOR, O. A.; BROSSARD, M.; RICHARD, G. Development and origin of the microgranular structure in Latosols of the Brazilian Central Plateau: significance of texture, mineralogy, and biological activity. Catena, Cremlingen, v. 76, n. 2, p. 122-134, 2009.

RESENDE, M.; CURI, N.; REZENDE, S. B.; CORRÊA, G. F. Pedologia: base para distinção de ambientes. Viçosa: NEPUT, 1995. 304 p.

RESCK, D. V. S.; FERRREIRA, E. A. B.; FIGUEIREDO, C. C.; ZINN, Y. L. Dinâmica da matéria orgânica no cerrado. In: SANTOS, G. A.; SILVA, L. S.; CANELLAS, L. P.; CAMARGO, F. A. O. Fundamentos da matéria orgânica do solo: ecossistemas tropicais e subtropicais. 2. ed. Porto Alegre: Editora Cinco Continentes, 2008. p. 359-417.

SALTON, J. C. Matéria orgânica e agregação do solo na rotação lavoura-pastagem em ambiente tropical. 2005. Tese (Doutorado em Agronomia/Ciência do Solo) - Universidade Federal do Rio Grande do Sul, Porto Alegre.

TISDAL, J. M.; OADES, J. M. Organic matter and water stable aggregates in soils. Journal of Soil Science, Oxford, v. 33, n. 2, p. 141-163, 1982.

VELASQUEZ, E.; PELOSI, C.; BRUNET, D.; GRIMALDI, M.; MARTINS, M.; RENDEIRO, A. C.; BARRIOS, E.; LAVELLE, P. This ped is my ped: visual separation and near infrared spectra allow determination of the origins of soil macroaggregates. Pedobiologia, Jena, v. 51, n. 1, p. 75-87, 2007.

VOLLAND-TUDURI, N. Nature et mode d'assemblage des constituintes minèraux et organiques dans des Ferralsols de la région des Cerrados (Brésil) evolution après mise em culture. $\mathrm{PhD}$-thesis. Université d'OrléansOrléans, France, 2005. Available at: <http:www. tel.archives-ouvertes.fr/docs/00/04/80/81/PDF/tel00009347.pdf.>. Accessed at: 7 ago. 2012.

YEOMANS, J. C.; BREMNER, J. M. A rapid and precise method for routine determination of organic carbon in soil. Communications in Soil Science and Plant Analysis, New York, v. 19, n. 13, p. 1467-1476, 1988. 